\title{
Hyperaccreting Disks around Neutrons Stars and Magnetars for GRBs: Neutrino Annihilation and Strong Magnetic Fields
}

\author{
Dong Zhang* and Z. G. Dai ${ }^{\dagger}$ \\ ${ }^{*}$ Department of Astronomy, The Ohio State University, 140W. 18th Ave., Columbus, OH, 43210, \\ USA \\ ${ }^{\dagger}$ Department of Astronomy, Nanjing University, Nanjing, 210093, China
}

\begin{abstract}
Hyperaccreting disks around neutron stars or magnetars cooled via neutrino emission can be the potential central engine of GRBs. The neutron-star disk can cool more efficiently, produce much higher neutrino luminosity and neutrino annihilation luminosity than its black hole counterpart with the same accretion rate. The neutron star surface boundary layer could increase the annihilation luminosity as well. An ultra relativistic jet via neutrino annihilation can be produced along the stellar poles. Moreover, we investigate the effects of strong fields on the disks around magnetars. In general, stronger fields give higher disk densities, pressures, temperatures and neutrino luminosity; the neutrino annihilation mechanism and the magnetically-driven pulsar wind which extracts the stellar rotational energy can work together to generate and feed an even stronger ultra-relativistic jet along the stellar magnetic poles.
\end{abstract}

Keywords: accretion: accretion disks — gamma rays: bursts - magnetic fields — neutrinos stars: neutron

PACS: $97.10 . \mathrm{Gz}, 98.70 . \mathrm{Rz}$

\section{INTRODUCTION}

The hyperaccreting disk surrounding a stellar-mass black hole possibly formed by the merger of a compact object binary or the collapse of a massive star has been argued to be a candidate for central engine of gamma-ray bursts (GRBs; e.g., Popham et al. 1999). On the other hand, both observational and theoretical evidences show that newborn neutron stars or magnetars rather than black holes can form in the GRB central engines (Usov 1992; Dai \& Lu 1998). However, all the previous magnetized neutron star or magnetar models ignore the accretion process which occurs onto a protoneutron star for the first several seconds. In the collapsar scenario, if the rotational core collapse can lead to the formation of a neutron star or a magnetar, it is possible that the prompt accretion or fallback process can make a hyperaccreting disk around the young formed star. Also a debris disk around a massive neutron star can be formed in compact binary mergers. Therefore we investigate the hyperaccreting neutron star or magnetar system, and show that it can also proposed as another possible central engine of GRBs (Zhang \& Dai 2008, 2009, 2010). For simplicity we consider the vertically integrated disk using the $\alpha$-prescription. To our knowledge, our work is the first to study the hyperaccreting disks around neutron stars possibly related to GRBs. 


\section{NEUTRINO-COOLED DISKS AROUND NEUTRON STARS}

In the hyperaccreting disk the Alfvén radius of the central star can be estimated as $r_{A} \simeq 0.207 \dot{M}_{-2}^{-2 / 7} M_{1.4}^{-1 / 7} \mu_{30}^{4 / 7} \mathrm{~km}$, where $\dot{M}=0.01 \dot{M}_{-2} M_{\odot} \mathrm{s}^{-1}$ is the accretion rate, $M=1.4 M_{1.4} M_{\odot}$ is the central star mass, $\mu=\mu_{30} 10^{30} \mathrm{G} \mathrm{cm}^{3}$ is the central magnetic flux. If the surface field $B_{0}$ is less than $B_{0} \leq B_{\text {crit }}=0.89 \times 10^{15} \dot{M}_{-2}^{1 / 2} M_{1.4}^{1 / 4} r_{*, 6}^{-5 / 4} \mathrm{G}$ with $r=r_{*, 6} 10^{6} \mathrm{~cm}$ being the star radius, the accretion flow will continue to be confined in the disk plane without co-rotating with the compact object or getting funneled onto the magnetar poles.

In the weak field case, the quasi-steady disk around a neutron star can be approximately divided into two regions - inner and outer disks, depending on the energy transfer and emission in the disk. For the outer disk, the heating energy rate $Q^{+}$is mainly due to local dissipation $\left(Q^{+}=Q_{\mathrm{vis}}^{+}\right)$, and the structure of the outer disk is very similar to the black hole disk. On the other hand, the heating energy in the inner disk includes both the energy generated by itself and the energy advected from the outer region $\left(Q^{+}=Q_{\mathrm{vis}}^{+}+Q_{\mathrm{adv}}^{+}\right)$, so the inner disk has to be dense with a high pressure. We take $Q^{+}=Q^{-}$and the entropy-conservation self-similar condition to describe the inner disk. The left panel of Figure 1 shows the size of the inner disk, which is determined by the global energy equation of the inner disk. Moreover, we also calculate the size of a low- $\alpha$ inner disk, which is smaller for a low accretion rate $\left(\leq 0.1 M_{\odot} \mathrm{s}^{-1}\right)$ compared to a high$\alpha$ disk $(\alpha \sim 0.1)$, and increases dramatically with increasing accretion rate. Also, the inner disk structure would not exist if the disk is unstable to drive a large scale thermal non-relativistic wind when the accretion rate $\geq 0.5 M_{\odot} \mathrm{s}^{-1}$.

We find that, due to the inner disk structure as well as the inner surface boundary of the compact star, the disk has a denser, hotter inner region with higher pressure compared to its black hold counterpart. Also, the entire disk can cool more efficiently via neutrino emission. The right panel of Figure 1 shows the total neutrino emission luminosity of the entire disk around a neutron star as a function of accretion rate, and we compare it with the neutrino luminosity from a black-hole disk. We see that the difference in neutrino luminosity between the neutron-star and black-hole cases is a strong function of the accretion rate. When the accretion rate is low, the total neutrino luminosity of the black-hole disk $L_{v, \mathrm{BH}}$ is much smaller than that of the neutron-star disk $L_{v, \mathrm{NS}}$, but $L_{v, \mathrm{BH}}$ and $L_{v, \mathrm{NS}}$ are similar for a moderate accretion rate.

\section{NEUTRINO ANNIHILATION}

Hyperaccreting black hole disks can convert some fraction of the net accretion energy into the energy of a relativistic jet by two general mechanisms: neutrino annihilation and magnetohydrodynamical (MHD) effects such as the Blandford-Znajek mechanism. However, for hyperaccretion disks surrounding neutron stars, the energy conversion mechanism is mainly due to the neutrino annihilation for the magnetic fields $\leq 10^{15}$ G. As the neutron star disk has a brighter neutrino luminosity compared with the black hole disk, the neutrino annihilation efficiency of the neutron star disk should be higher than that of the black hole disk. Moreover, the surface boundary of the neutron 

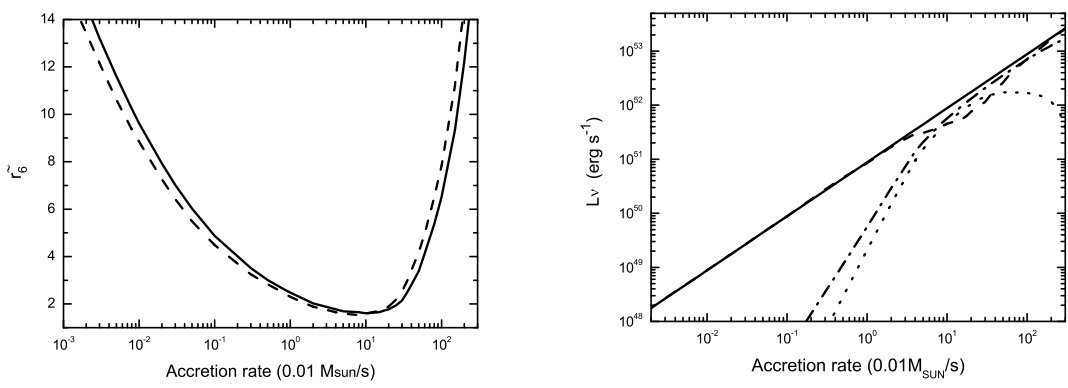

FIGURE 1. (a) Left panel: the radius $r /\left(10^{6} \mathrm{~cm}\right)$ between the inner and the outer disks with the center neutron star $M=1.4 M_{\odot}$ (solid line), and $M=2.0 M_{\odot}$ (dashed line). (b) Right panel: Neutrino luminosity from the disk with $M=1.4 M_{\odot}$. The solid line corresponds to the maximum energy release rate of the disk around a neutron star, the dashed line to the neutrino luminosity from the inner disk, the dotted line to the neutrino luminosity from the outer disk, and the the dash-dotted line to the neutrino luminosity from a black hole disk.
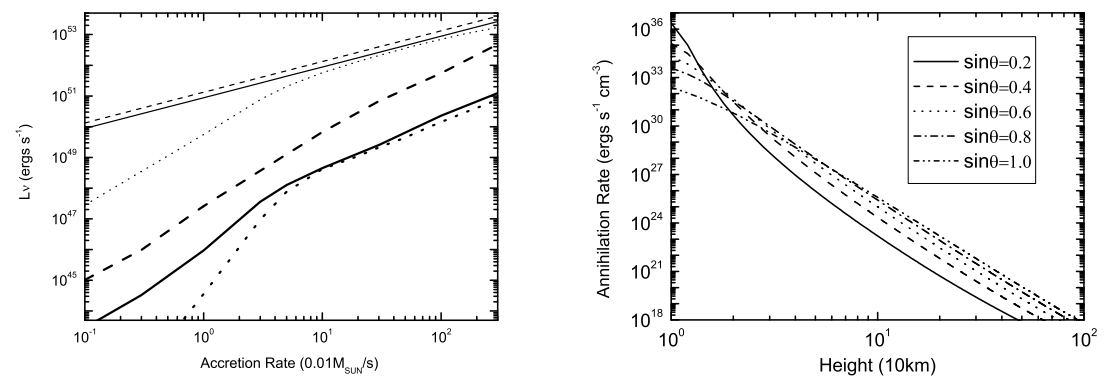

FIGURE 2. (a) Left panel: Neutrino annihilation luminosity $L_{v \bar{v}}$ (thick lines) and total neutrino emission luminosity $L_{v}$ (thin lines) as functions of accretion rate. The solid lines correspond to the neutron star disk with the boundary layer emission efficiency $\eta_{s}=0$, the dashed lines to $\eta_{s}=0.5$, and the dotted lines to the black hole disk. (b) Right panel: The neutrino annihilation rate $l_{v \bar{v}}$ along the stellar magnetic pole as a function of height for accretion rate $\dot{M}=0.5 M_{\odot} \mathrm{s}^{-1}$ in strong field case, where $\sin \theta \simeq \sqrt{r_{*} / r_{A}}$ measures the strength of funnel accretion, smaller $\sin \theta$ corresponds to stronger field and funnel accretion.

star, which carries away gravitational-binding energy by neutrino emission makes the neutrino annihilation luminosity of the neutron star disk be even higher. We follow the approximate method used by Popham et al. (1999) to calculate the annihilation rate, and we introduce the efficiency factor $\eta_{s}$ to measure the energy emitting from the stellar surface as $L_{s} \simeq \eta_{s} G M \dot{M} /\left(4 r_{*}\right)$ with $r_{*}$ being the neutron star radius.

The left panel of Figure 2 shows the total neutrino annihilation luminosity and the emission luminosity of a neutron-star disk with different surface boundary layer conditions $\left(\eta_{s}=0\right.$ and 0.5$)$. The annihilation luminosity of the neutron-star disk are brighter than those of a black hole disk with the same mass and accretion rate. If we study the neutrino annihilation from the entire disk without surface boundary emission $\left(\eta_{s}=0\right)$, the difference between $L_{v \bar{v}, N S}$ and $L_{v \bar{v}, B H}$ is more significant for a low accretion rate 
than for a high accretion rate. On the other hand, neutrino emission from the neutron star surface boundary layer $\left(\eta_{s}=0.5\right)$ makes the annihilation luminosity be more than one order of magnitude higher than that without boundary emission $\left(\eta_{s}=0\right)$. Therefore, a lower-spin neutron star (larger $\eta_{s}$ ) with hyperaccreting disk around it could have an obviously higher annihilation efficiency than that of a higher-spin neutron star.

\section{EFFECTS OF STRONG MAGNETIC FIELDS}

Strong fields $\geq 10^{15} \mathrm{G}$ from the center magnetar can play a significant role in affecting the disk properties and even changing the accretion process (Zhang \& Dai 2010). We consider the magnetar field has a dipolar vertical component $B_{z}$. The differential rotation between the disk and the magnetar will generate a toroidal field component $B_{\phi}$ and a relatively weak radial component $B_{r}$. The generated field can have an open or closed configuration, depending on the disk's viscous turbulence, magnetic diffusivity and disk angular velocity. For the strong field environment, the quantum effects (Landau levels) and large scale field coupling play two competitive roles in changing the disk properties, the former to decrease the pressure, density and neutrino luminosity with increasing field strength, while the latter to increase them. However, in most cases the field coupling is more significant than the microphysical quantum effect. Note that in the hyperaccreting disks, the funnel accretion can only be important for extremely strong fields. The funnel flow will cover a ring-like belt of "hot spot" around the magnetar surface and emit thermal neutrinos. Because of the more concentrated emission from the "hot spot", the funnel accretion can accumulate even more powerful neutrino annihilation luminosity than the weak field case (see right panel of Figure 2).

The neutrino annihilation process both from the magnetar surface and from the disk plane will be higher than that without fields. Moreover, the neutrino annihilation mechanism and the magnetic activity from the stellar surface (i.e., the pulsar wind mechanism) can work together to generate and feed an ultra-relativistic jet along the stellar magnetic poles. If the stellar spin period is sufficiently short (e.g., $\sim 4 \mathrm{~ms}$ for the field $\sim 10^{16}$ $\mathrm{G}$ and $\dot{M}=0.1 M_{\odot} \mathrm{s}^{-1}$ ), the jet from the magnetar will be magnetically-dominated and mainly feeded by extraction the stellar rotational energy. If the magnetar spin period is long, the jet is thermally-driven and feeded by the annihilation process. In this case, an energetic ultrarelativistic jet via neutrino annihilation can be produced above the stellar polar region if the disk accretion rate and the stellar surface luminosity are sufficiently high. In the intermediate case, on the other hand, the relativistic jet can be launched by the pulsar-wind-like process and neutrino annihilation together.

\section{REFERENCES}

1. Dai, Z. G., \& Lu, T. 1998b, Phys. Rev. Lett., 81, 4301

2. Popham, R., Woosley, S. E., \& Fryer, C. 1999, ApJ, 518, 356

3. Usov, V. V. 1992, Nature, 357, 472

4. Zhang, D., \& Dai, Z. G. 2008, ApJ, 683, 329

5. Zhang, D., \& Dai, Z. G. 2009, ApJ, 703, 461

6. Zhang, D., \& Dai, Z. G. 2010, ApJ, 718, 841 